\title{
EL TREKKING EN EL TRAYECTO FORMATIVO DE PROFESORES DE EDUCACIÓN FÍSICA: INTEGRANDO EL ENSEÑAR, APRENDER Y ENSEÑAR A ENSEÑAR EN LA FORMACIÓN PROFESIONAL
}

\author{
THE TREKKING DURING PHYSICAL EDUCATION IN THE WAY THE TRAINING OF \\ TEACHERS: INTEGRATING THE TEACHING, LEARNING AND TEACHING TO TEACH \\ PROFESSIONAL FORMATION
}

\begin{abstract}
Reising, Maria Lilén \& Alder, Inés
Departamento de Educación Física, Centro Regional Universitario Bariloche. Universidad Nacional del Comahue, República Argentina.
\end{abstract}

REISING M.L. \& ALDER I. (2014). El trekking en el trayecto formativo de profesores de educación física: integrando el enseñar, aprender y enseñar a enseñar en la formación profesional. Mot. Hum. 15(1): 27-33.

\section{RESUMEN}

El presente artículo se enmarca en el proyecto de investigación "El andinismo en la Educación Física: seguridad, enseñanza y formación docente” (B169) desarrollado en el Centro Regional Universitario Bariloche dependiente de la Universidad Nacional del Comahue. Dicho proyecto toma como principal foco de estudio las asignaturas del Profesorado de Educación Física vinculadas con la Orientación Actividades de montañismo y escalada a fin de indagar sobre los procesos de enseñanza y aprendizaje que en ellas se llevan a cabo. En esta oportunidad se comparten avances relacionados con el trekking y su enseñanza en la formación de Profesores de Educación Física. Se analizan programas de cátedra y entrevistas realizadas a profesores, asistentes y ayudantes con el objetivo de identificar propósitos, estrategias de enseñanza, secuencia de contenidos y selección de itinerarios que promuevan en el estudiante la apropiación de la actividad, el conocimiento del medio en el cual se realiza y la transferencia de contenidos a otros contextos educativos.

A partir de la revisión de marcos teóricos se define conceptualmente al trekking e identifican variables que otorgan rasgos particulares al desarrollo de la actividad. Se establecen categorías que denotan grados de complejidad a fin de visualizar y establecer criterios para la elección de opciones posibles de ser implementadas de manera segura y responsable en distintos ámbitos de la educación formal y no formal.

Palabras Clave: Trekking - Montañismo - Formación docente - Educación física

\section{ABSTRACT}

This article is part of the research project "The andinism in Physical Education: safety, education and teacher training" (B169) developed at the Centro Regional Universitario Bariloche dependant of the Universidad Nacional del Comahue. The project has its main focus of study on the subjects in the Faculty of Physical Education related to climbing and mountaineering orientation, to investigate the processes of teaching and learning that take place. On this opportunity, advances related to trekking and its teaching in the training of physical education teachers are shared. Academic programs and interviews with teachers, assistants and aides are analyzed with the objective of identifying purposes, teaching strategies, contents sequences and choice of itineraries that promote in the student the appropriation of the activity, the knowledge of the environment in which is performed and the transference of contents to other educational contexts. From the review of theoretical frameworks "the trekking" is conceptually defined and variables that give particular features to the development of the activity are identified. Categories indicating degrees of complexity to visualize and establish criteria for the choice of possible options to be implemented safely and responsibly in different areas of formal and non-formal education are established.

Keywords: Trekking - Mountaineering - teacher training - physical education 


\section{INTRODUCCIÓN}

Las actividades en contacto con la naturaleza y específicamente aquellas vinculadas con el montañismo han sumado gran cantidad de adeptos en los últimos tiempos. No solo se las ha considerado como una alternativa más para el uso del tiempo libre sino que también han sido incorporadas en diferentes diseños curriculares escolares debido al potencial educativo que ofrecen las mismas.

Pensar en la inclusión de la actividad del trekking en el campo de la Educación Física necesariamente nos lleva a abordar de manera conceptual el tema, caracterizar la actividad y establecer categorías que ayuden al profesional a planificar secuencias didácticas posibles de llevar a cabo en distintos contextos educativos. En este sentido, autores como Arribas (2008), Sáez y Giménez (2005) realizan un análisis de las actividades en el medio natural aplicado al caso de España, en el cual describen la conformación de los contenidos de actividades en la naturaleza en el profesorado de Educación Física y las competencias profesionales para desarrollar la labor como docentes de las mismas.

Algunas preguntas que surgen son: ¿Qué es el trekking? ¿Qué implica enseñar a caminar en la montaña? ¿Con qué conocimientos debe contar el profesional para llevarla a cabo?, ¿De qué manera se prepara a los futuros docentes en Educación Física para el desafío de trabajar en un medio naturalmente cambiante, escenario de múltiples posibilidades?, entre otras.

\section{¿A qué nos referimos cuando hablamos de trekking?}

En la actualidad, la palabra trekking es utilizada en distintos ámbitos para referirse prácticamente a cualquier actividad que consista en caminar por el medio natural. En este sentido, su uso pareciera no quedar restringido exclusivamente a aquellas caminatas que se realizan en el contexto particular de la montaña sino que se amplía a otros espacios tanto urbanos como periurbanos, desvirtuando de esta manera las características propias e identitarias de la actividad.
Teniendo en cuenta los aportes de Zorrilla (2001) y Moscoso (2003) está claro que el origen de la palabra se vincula directamente a una actividad que consiste en "caminar por la montaña”, es así que proponemos una aproximación terminológica de dicho concepto a fin de establecer categorías que ayuden a comprender las características que puede adquirir el mismo (Alder y Reising, 2013).

El medio natural en general y la montaña en particular es entendido como aquel espacio de la naturaleza que hace de soporte de una amplia gama de prácticas reconocidas "de aventura", entre ellas el trekking. Como denominador común todas ellas se realizan en un medio inestable cargado de incertidumbre debido a su carácter cambiante. Dicha incertidumbre, entendida como la característica que hace que una cosa, circunstancia o situación no pueda ser previsible ni anticipada, en estas prácticas se vincula fundamentalmente a dos tipos de factores: a) ambientales: lluvias, nieve, cambios en cobertura del terreno, crecidas de ríos y arroyos, cambio del tiempo, entre otros y b) factores personales: están ligados a la experiencia, al nivel de ejecución de la acción motriz, a la capacidad de adaptabilidad y a su gestión del riesgo. Pueden ser modificados o anticipados por el sujeto.

La incidencia y combinación de estos factores le imprimen al trekking características particulares. A fin de clarificar los rasgos que adquiere la actividad, se comparten categorías que intentan mostrar distintos niveles de realización, posibles de tener en cuenta al momento de organizar la enseñanza. A partir de lo dicho se distingue entre: a) el trekking sin dificultad técnica (TSDT ) y b) el trekking con dificultad técnica (TCDT).

\section{Trekking sin dificultad técnica}

Esta categoría la integran aquellas actividades que consisten en desplazarse caminando por terreno montañoso y que en cuyo recorrido no requieran de la utilización de equipo técnico, técnicas avanzadas y miembros superiores para estabilizarse durante el desplazamiento. El tránsito se realiza generalmente por senderos o picadas diseñados para tal fin cuyo punto de inicio puede o no coincidir con el punto final. Durante el recorrido se suelen observar señalizaciones (pintadas en árboles, piedras, torres 
de piedras, cartelería, etc.) que indican el camino correcto a seguir. Pueden tener una duración variable como así también realizarse en cualquier época del año.

Tomando como referencia la categorización de senderos del Parque Nacional Nahuel Huapi (Disposición $\mathrm{N}^{\circ} 590 / 08$ ), se distinguen dos subcategorías: 1) el trekking sin dificultad técnica grado de complejidad baja "fácil": para el cual no se requiere de experiencia ni de preparación física. Dentro de este grupo entran aquellas opciones que más allá de la época del año no varían su dificultad. Por lo general tienen una duración que no excede la media jornada. El terreno evidencia ser suelo firme, llano o de pendiente moderada, y 2) trekking sin dificultad técnica grado de complejidad "media": para este caso se requiere de una mínima experiencia y preparación física. La gran mayoría de los senderos contemplados dentro de esta clasificación varían su dificultad según la época del año, quedando por lo general encuadrados en dificultad media únicamente durante el período estival. Al igual que en la subcategoría anterior, el sendero está bien definido aunque puede presentar zonas de mayor pendiente y tratarse de superficies variables, inestables ó desparejas según su localización. La duración puede o no superar la jornada. Se piensa como máximo que esta subcategoría no exceda los dos días de caminata.

\section{Trekking con dificultad técnica}

En esta categoría entran aquellas actividades que consisten en desplazarse caminando por terreno montañoso y en cuyo recorrido se requiere de la utilización de técnicas específicas y/o equipo técnico para avanzar de manera responsable y segura.

Autores como Weineck (2005), entienden a la técnica como el conjunto de procesos desarrollados generalmente por la práctica para resolver de manera más racional y económicamente un problema motor determinado. En el trekking, la técnica no es exclusiva a la necesidad de utilizar equipo específico sino que además se la requiere con mayor intensidad en la medida que el caminar aumenta su complejidad debido fundamentalmente a las características del terreno, tipo de cobertura, pendiente / desnivel y su combinación con las variables en función de la época del año. Se aclara asimismo que si bien el tramo de "dificultad" para el cual se requiera otro tipo de experiencia pueda ser corto en relación al recorrido total del trekking, ya es condición suficiente como para clasificarlo dentro de esta categoría, es decir, las clasificaciones propuestas tienen en cuenta las características de la totalidad del recorrido y es a partir de la dificultad máxima y/o grado de pendiente que se lo distingue en una u otra. En este sentido, el terreno por el cual se transita puede presentar pendientes que superen $\operatorname{los} 25^{\circ}-30^{\circ}$ y requerir de miembros superiores para estabilizarse. Frecuentemente la definición del recorrido es elegido y diagramado por quien realiza la actividad ya que puede no encontrarse señalizado con la misma precisión que en aquellos trekking encuadrados en la categoría TSDT. Un ejemplo de ello se da al superar el límite de vegetación, tratándose mayoritariamente de desplazamientos por pedreros no estables donde no sólo se está en presencia de situaciones expuestas sino que además se observan cambios notables en el estado del tiempo. La duración de la actividad suele ser mayor que los trekking sin dificultad técnica aunque no es una variable principal que evidencie la distinción entre un nivel y otro de complejidad.

En esta categoría, según la categorización de senderos del Parque Nacional Nahuel Huapi se distinguen dos subcategorías: a) el trekking con dificultad técnica complejidad "alta": se requiere de experiencia en senderos de montaña y una buena condición física. El terreno por lo general es inestable, se trata en su mayoría de desplazamientos por pedreros y/o superficies cubiertas por nieve. Para estas últimas se requiere además contar con conocimientos sobre nivología. Frecuentemente la elección de itinerario está definida por la orientación de quien la práctica y la lectura de la topografía, no obstante pueden observarse marcas o señalizaciones distantes y b) el trekking con dificultad técnica con grado de complejidad "muy alta": la señalización es escasa o nula, debido a ello el camino a seguir es determinado mayoritariamente por quien realiza la actividad. Suele presentar tramos técnicos donde se requiera la utilización de equipo adicional así como también el conocimiento de nivología en época invernal a fin de poder 
discernir sobre la posibilidad de avalanchas. En este sentido, la incertidumbre pareciera tener mayor intensidad. La duración puede ser de varias jornadas. En caso de considerar no contar con los conocimientos necesarios para desenvolverse de manera autónoma en este tipo de situación se sugiere realizar la actividad en compañía de expertos (guías profesionales habilitados).

Para ambas categorías (TSDT y TCDT), una forma particular que puede adquirir el trekking es la de travesía. La misma consiste en realizar una caminata por la montaña cuyo recorrido atraviesa uno o varios cordones montañosos. El punto de inicio puede no coincidir con el punto final de destino, requiriendo para su realización exitosa de una mayor logística en su preparación previa. Suelen tener una duración superior a la jornada, pudiendo extenderse a varios días (López, Palacio y Reising 2012).

\section{El trekking como contenido en el trayecto formativo de profesores de Educación Física}

El plan de estudio del profesorado en Educación Física del Centro Regional Universitario Bariloche complementa la formación tradicional con asignaturas relacionadas al contexto regional, entre ellas el trekking, la escalada en roca, la escalada deportiva en muro artificial y el esquí (Fernández, López 2010). Centraremos la atención en la primera de ellas a fin de compartir el modo en que se aborda la enseñanza con futuros formadores en actividades regionales de montaña.

Partiendo de un diagnóstico inicial, se advierte que la mayoría de los estudiantes que ingresan al profesorado manifiestan tener escasa experiencia a nivel grupal y educativo en actividades en contextos de montaña. Reconocer dicha situación hace pensar en cómo plantear la enseñanza de contenidos vinculados a las actividades de montañismo a fin de brindar multiplicidad de vivencias y experiencias en el terreno que le faciliten al estudiante tender relaciones entre el aprender a caminar en el contexto de la montaña y el aprender a enseñar el trekking (Alder, Reising 2013).

La orientación Actividades de Montañismo y Escalada comienza en el segundo año de la carrera.
Las prácticas que se realizan de manera inicial abordan el trekking de manera introductoria brindando los conocimientos básicos para desenvolverse en el terreno. En esta primer instancia el docente es quien planifica, decide el itinerario y conduce al grupo con el propósito de acercar la actividad al estudiante de manera gradual y progresiva, favorecer la formación integral incorporando nuevas posibilidades motrices y el desarrollo de su corporeidad, su relación con el grupo y el entorno. Los trekking que aquí se realizan están encuadrados mayoritariamente en la categoría TSDT.

En tercer y cuarto año se enfatiza en el desarrollo de la autonomía del sujeto, en sus posibilidades de adaptabilidad en el medio y la incorporación adecuada de la administración como gestores de riesgo. Las dos asignaturas que abordan la temática proponen una secuencia de contenidos que en cada año son abordados con mayor profundización aplicando los mismos de manera práctica a distintas situaciones climatológicas y diversidad de terrenos. En este sentido, los estudiantes van comprendiendo e integrando diferentes aspectos relacionados con la planificación y organización de una salida para luego llevarla a cabo. En esta instancia, se abordan actividades de trekking con dificultad técnica en su mayoría de complejidad alta.

Al analizar extractos de entrevistas realizadas a docentes de la Orientación Actividades de montañismo y escalada se identifican dos grandes grupos de contenidos:

a) aquellos que son estipulados en el plan de estudios, los programas de cátedra y las planificaciones: el equipo personal y grupal, orientación, ritmo de marcha, planificación, seguridad, entre otros y b) aquellos que surgen durante la práctica a partir de la combinación grupo, actividad, lugar y condiciones meteorológicas. Estos últimos están relacionados con la seguridad y prevención, el caminar por el medio y su técnica, la selección del itinerario a partir de lo que el medio nos muestra, la orientación en terreno, el grupo en la montaña y su interrelación, entre otros.

Algunos fragmentos de entrevistas dan cuenta lo explicitado anteriormente: "La enseñanza se 
plantea a partir de una progresión desde caminatas más simples de un día, dos días, distintos tipos de terrenos ya sea la estepa el bosque, alta montaña refugios, o sea tiene una serie de contenidos en esa planificación anual que van con distinta complejidad...”, “...es una orientación que necesita de terreno para aprenderlo y poder tener las herramientas, la comodidad y la tranquilidad que hace falta para desplazarte con un grupo...”, “...en un principio uno hace hincapié en la movilidad en el medio, en la elección del itinerario y en la conducción de grupo, que se continúa con todo eso en las tres materias..." "la parte de cartografía, todo lo que sería la navegación terrestre, con mapa y con brújula...van de la mano de la práctica", "Hay algunos contenidos técnicos que realmente hay que explicarlos, hablarlos y ponerlos en práctica, vivenciarlos de manera directa para poder aprenderlos...". Puede apreciarse que aprender la actividad implica además aprender acerca del medio y uno mismo en relación a ambos. Estos aprendizajes se plantean de manera gradual con la articulación espiralada en forma dialéctica de contenidos teórico-prácticos. En esta instancia se pretende realizar la mayor cantidad de situaciones vivenciales en terreno.

“... Te vas a encontrar con un montón de situaciones imponderables que resolver: resolver una situación meteorológica, los ritmos de marcha con 20 compañeros...". "Hacer trekking solo no es lo mismo que hacer trekking en grupo, los contenidos van surgiendo a partir de lo que nos vamos encontrando en el medio y de la relación grupo, actividad, medio natural....", "damos bases generales tanto sea de contenido como de terreno para que el alumno tenga un poder de discernimiento sobre qué es lo que está decidiendo, cuales son las herramientas que puede utilizar, donde debe ir, donde no debe estar, y en tal caso las herramientas para poder actuar sobre cosas que no están en su planificación pero sí que le pueden pasar...". Se observa que los docentes manifiestan la importancia de atender a contenidos que atraviesan la práctica más allá de la temática prevista en la planificación. La mayoría de ellos coincide en que el abordaje de los mismos responde a lo que surge en el entorno, en el grupo y la relación entre ambos: "Si no estoy en el medio no puedo decirte bien qué es todo lo que finalmente voy a hacer ni cómo..”. “...no está muy pautado, es medio abierto, eso está bueno porque en la naturaleza se dan cosas muy circunstanciales, espontáneas y hay que saber interpretar esto y aprovecharlo para transmitir conocimientos".

Tal situación coincide de alguna manera con lo expresado por Guillen, Lapetra y Casterad (2000) como factores incontrolables del medio, aludiendo al carácter cambiante del mismo y a la incertidumbre que generan las prácticas educativas en dicho contexto. Es decir, en gran parte la interacción grupo-medio-actividad va marcando qué, cómo y cuándo enseñar. Esta situación evidencia que la enseñanza en la montaña se da más allá de una planificación original. Por tanto, desempeñarse profesionalmente en el medio natural y en la montaña en particular implica poder captar lo que el medio nos presenta, enriqueciendo así la propuesta pedagógica. En este sentido, el carácter áulico de la montaña se distingue de otros espacios en cuanto a su nivel de previsión y estructuración de la planificación (Reising, 2011).

La montaña ofrece variedad de escenarios que requieren de una permanente adaptación. Aún cuando se sabe que "el caminar" es una habilidad motora básica (Ruiz Pérez 1993), realizar dicha habilidad en diferentes terrenos, en situaciones nuevas, más complejas y desconocidas, incrementa la incertidumbre y la adaptabilidad de su motricidad pasa a depender también de otros factores como los climáticos. Mediante la influencia de ellos se presenta la imprevisibilidad de los cambios físicos del terreno requiriendo por parte de quienes transitan la montaña un proceso de adaptación permanente y de incorporación de nuevos aprendizajes. El sujeto que realiza este tipo de actividades en el medio natural y

sobre todo las actividades vinculadas a la práctica del montañismo además de adquirir otras habilidades para transitarla comienza a establecer relaciones más complejas entre "sujeto-medio natural y actividad" que lo orientará en sus formas de actuar encaminado a superar las diversas situaciones que se presentan durante la misma. “...la adaptación psicomotriz que uno realiza al pasar de un plano a otro más inclinado, por terrenos que tienen diferente tipo de textura, 
posición, estabilidad es algo que no se puede aprender de los libros.... se requiere readaptar el sentido del equilibrio, el tono muscular, la utilización de los músculos..." “... al principio es como volver a aprender a caminar, conocer el propio esfuerzo y las propias capacidades, es un proceso que no es instantáneo y de ninguna manera se aborda desde la teoría". Los docentes dejan en evidencia la importancia de generar estímulos motrices y diversidad de vivencias en el terreno para posibilitar la incorporación de nuevas habilidades y destrezas motrices para transitar en forma autónoma y segura por el medio natural. Esta competencia motriz, definida por Ruiz Pérez (1993/2001) como un proceso dinámico y complejo, se caracteriza por una sucesión de progresos y cambios motrices de los sujetos y las acciones con los otros y los objetos. Podemos decir que los estudiantes van construyendo la misma de manera particular pero también se ve influenciada en este caso por factores intrínsecos, es así que aunque se presente el mismo estímulo vivencial a todos los sujetos lo que cada sujeto percibe depende de sus intereses, actitudes, experiencias previas y estructuras cognitivas (Woolfok 1984).

\section{Consideraciones finales}

La montaña se constituye en un espacio particular, complejo, que crea incertidumbre. Pensamos que el incorporar al trekking como contenido en la formación de los futuros profesores se constituye en un gran aporte al desarrollo del campo de la Educación Física. Cada vez son más frecuentes las propuestas relacionadas con la temática en ámbitos educativos formales y no formales debido a los valiosos aportes que la actividad puede promover en el desarrollo integral de los sujetos, así como por su influencia posterior en la realización personal del adulto (Moscoso y González 2004).

En relación al proceso pedagógico desarrollado en el contexto de formación de formadores se visualiza la necesidad de enseñarle al estudiante el cómo desenvolverse en la montaña. La seguridad es comprendida como un punto clave a aprender así como también la lectura de los indicios que el medio presenta. Ambos se constituyen como determinantes del nivel de riesgo. En este sentido se enfatiza la importancia de tener una mirada amplia y flexible al momento de organizar y planificar propuestas pedagógicas en dicho contexto.

En los relatos de los docentes se explicita con claridad la necesidad de contar con un espacio de formación específica sobre la temática para llevar a cabo actividades educativas en la montaña. La propuesta curricular de las cátedras que integran contenidos transversales en forma espiralada a lo largo de la especialización de los estudiantes testifican la importancia de prestar cierta atención en la formación inicial de los mismos.

Se destaca la vivencia en el medio a través de la experiencialidad y adaptabilidad, factores determinantes para ser un buen gestor de riesgo y la necesidad de generar instancias de capacitación y actualización permanente.

En el intento de definir conceptualmente al Trekking se distinguen categorías que denotan grados de complejidad. Nos surgen algunas preguntas: ¿Cómo lograr mayor precisión en la clasificación propuesta al combinar factores relacionados con el sujeto y el medio? ¿Se podrían encontrar otras variables que ayuden a definir estas categorías? ¿Cuál es el límite entre un nivel de complejidad y otro? ¿Hay alguna/s variable/s que merezca tener mayor relevancia por sobre otra/s al momento de establecer la distinción conceptual?

\section{REFERENCIAS BIBLIOGRÁFICAS}

Alder M.L. y Reising I. (2013). Construyendo puentes entre el caminar y el aprender a enseñar el trekking. Ponencia presentada en el VI Congreso Nacional y IV Internacional de investigación educativa. "La investigación educativa en el contexto latinoamericano" Universidad Nacional del Comahue. Facultad de Ciencias de la Educación.

Arribas C. (2008). El pensamiento y la biografía del profesorado de Actividad Física en el medio natural. Un estudio multicaso en la formación universitaria orientado a la comprensión de modelos formativos". Universidad de Valladolid. Facultad de Educación y Trabajo Social.

López E. y Fernández M. (2011) Las actividades de Andinismo en la formación del Profesorado en Educación Física. Revista EFEI 1(1), 34-38.

Guillén, R., Lapetra, S. y Casterad, J. (2000). Actividades en la Naturaleza. Barcelona:

INDE. 


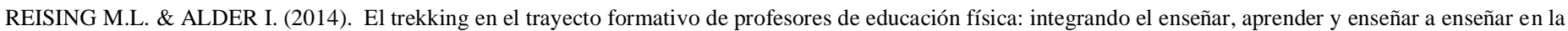
formación profesional. Mot. Hum. 15(1): 27-33.

López, Palacio y Reising (2012). Las caminatas en la montaña y la formación docente en el Profesorado de Educación Física, CRUB-UNCo. Ponencia presentada en las III $^{\circ}$ Jornadas de Investigación, Facultad de Educación Física. Departamento de investigación FACDEF.

Moscoso Sánchez, David (2003). La montaña y el hombre en los albores del Siglo XXI, España, Barrabés.

Moscoso S. D. y González F.M.T. (2004). La montaña como observatorio de lo social. IESA Working Paper Series WP 2104.

Parque Nacional Nahuel Huapi. Disposición N ${ }^{\circ} 590 / 08$ : Categorización de senderos y circuitos del Parque Nacional Nahuel Huapi.

Reising, M. L. (2011) "Algunas reflexiones sobre el proceso de enseñanza y aprendizaje de las actividades recreativas de montaña en ámbitos educativos no formales". Ponencia presentada en el 9 Congreso Argentino y 4 Latinoamericano de Educación Física y Ciencias. Universidad Nacional de La Plata, junio de 2011.

Ruiz P. L.M. (1993) "Desarrollo motor y actividades físicas". Edit. Gymnos. España.

Ruiz P. L.M. (2001) "Desarrollo, comportamiento motor y deporte”. Editorial Síntesis, España.

Sáez Padilla, Giménez Fuentes Guerra (2005) "Análisis de la oferta formativa en actividades en el medio natural", http://www.efdeportes.com/ Revista Digital - Buenos Aires Año 10 - $\mathrm{N}^{\circ} 83$.

Weineck, J. (2005). Entrenamiento Total. Editorial Paidotrubo. Barcelona.

Woolfok, A. E. y McCune, L. (1984). Psicología de la Educación. Madrid: Narcea.

Zorrilla, J. (2001). Diccionario de los deportes de montaña. España, Desnivel.

\section{Dirigir Correspondencia a:}

María Lilén Reising

Email: lilenreising@gmail.com

Fono: 0294-4610438

Quintral 1250, Centro Regional Universitario

Bariloche (CRUB).

Bariloche, Rio Negro. Argentina

\section{RECIBIDO: 20-06-2014}

\section{ACEPTADO: 30-06-2014}

